\title{
Analysis of Carbon Nanotubes on the Mechanical Properties at Atomic Scale
}

\author{
Xiaowen Lei, ${ }^{1}$ Toshiaki Natsuki, ${ }^{2}$ Jinxing Shi, ${ }^{1}$ and Qing-Qing Ni ${ }^{2,3}$ \\ ${ }^{1}$ Interdisciplinary Graduate School of Science and Technology, Shinshu University, 3-15-1 Tokida, Ueda, Nagano 386-8567, Japan \\ ${ }^{2}$ Department of Functional Machinery and Mechanics, Shinshu University, 3-15-1 Tokida, Ueda, Nagano 386-8567, Japan \\ ${ }^{3}$ Institute of Road and Bridge Engineering, Dalian Maritime University, 1 Linghai Road, Ganjingzi, Dalian 116026, China
}

Correspondence should be addressed to Toshiaki Natsuki, natsuki@shinshu-u.ac.jp

Received 14 June 2010; Accepted 23 August 2010

Academic Editor: Junping Wang

Copyright (C) 2011 Xiaowen Lei et al. This is an open access article distributed under the Creative Commons Attribution License, which permits unrestricted use, distribution, and reproduction in any medium, provided the original work is properly cited.

\begin{abstract}
This paper aims at developing a mathematic model to characterize the mechanical properties of single-walled carbon nanotubes (SWCNTs). The carbon-carbon $(\mathrm{C}-\mathrm{C})$ bonds between two adjacent atoms are modeled as Euler beams. According to the relationship of Tersoff-Brenner force theory and potential energy acting on $\mathrm{C}-\mathrm{C}$ bonds, material constants of beam element are determined at the atomic scale. Based on the elastic deformation energy and mechanical equilibrium of a unit in graphite sheet, simply form ED equations of calculating Young's modulus of armchair and zigzag graphite sheets are derived. Following with the geometrical relationship of SWCNTs in cylindrical coordinates and the structure mechanics approach, Young's modulus and Poisson's ratio of armchair and zigzag SWCNTs are also investigated. The results show that the approach to research mechanical properties of SWCNTs is a concise and valid method. We consider that it will be useful technique to progress on this type of investigation.
\end{abstract}

\section{Introduction}

Since the discovery by Iijima in 1991 [1], carbon nanotubes (CNTs) have generated huge activities in most areas of science and engineering due to their unprecedented mechanical, electrical, and thermal properties [2-13]. Especially in mechanical field, experimental measurements have determined that CNTs possess excellent mechanical properties [14-21]. Therefore, an effective method to analyze the basic characteristics of nanosized CNTs is essential.

In the past, researchers used experimental method to measure mechanical properties of CNTs. Treacy et al. [14] firstly measured the amplitude of intrinsic thermal vibrations observed in transmission electron microscopy (TEM). The average value of Young's modulus of CNTs derived from this experimental technique is $1.8 \mathrm{TPa}$ by 11 tubes, in which the lowest value and the highest value are $0.40 \mathrm{TPa}$ and $4.15 \mathrm{TPa}$, respectively. Later, Poncharal et al. [18] obtained Young's modulus of CNTs which is between 0.7 and $1.3 \mathrm{TPa}$ by electromechanical resonant vibrations. In addition, based on an atomic force microscope (AFM), Wong et al. [20] in 1997 firstly directly measured the stiffness constant of armchair
MWCNTs pinned at one end, from which the value of Young's modulus of CNTs is $1.28 \mathrm{TPa}$. Salvetat et al. [21] used the AFM for experiment of bending an armchair (multiwalled carbon nanotubes) MWCNT pinned at each end over a hole and obtained an average modulus value of CNTs of $0.81 \mathrm{TPa}$. These experiments all promote the research of mechanical properties of CNTs. However, in description of nanoscale structures, the results are with experimental errors.

Meanwhile, for researching mechanical properties of CNTs, a number of researchers solved the difficulties in nanosized experiments in terms of computer simulation. For the analysis of nanostructural materials, atomic simulation methods such as first-principle quantum-mechanical methods [22], molecular dynamics (MD) [23, 24], and Monte Carlo [25] simulations have been routinely adopted. As early as 1993, Overney et al. [26] calculated Young's modulus of rigid short SWCNTs which is $1.5 \mathrm{TPa}$, approximately equal to that of graphite. This was followed by a range of papers predicting that Young's modulus of CNTs is close to $1 \mathrm{TPa}$ independent of type and diameter [27]. Yakobson et al. [28] fitted these results by MD simulations of the continuum shell 
model. Unlike the previous work that assumed a thickness of $0.34 \mathrm{~nm}$, both the thickness and Young's modulus were taken as the fitting parameters, yielding a thickness of $0.066 \mathrm{~nm}$ and Young's modulus of $5.5 \mathrm{TPa}$. The MD approach was also used by $\mathrm{Lu}[27,29]$ who reported that Young's modulus is $1 \mathrm{TPa}$ and claimed that chirality and the number of walls have little effect on the value of Young's modulus. A different potential model was used by Yao and Lordi [16] who obtained Young's modulus of CNTs as $1 \mathrm{TPa}$. Although MD method has been widely used in simulating the properties of nanostructural materials, it is complex and time consuming, especially for large amount atomic systems. Therefore, the continuum mechanics seems to be a better way to investigate the properties of CNTs. However, for the case of nanoreinforced adhesives, these models cannot accurately describe the influence of the relationship between carbon atoms upon the mechanical properties and their interactions in the composite systems because they lack the appropriate constitutive relations that govern material behavior at this scale [30]. Another modeling approach is the atomic-based continuum technique, which has the unique advantage of describing atomic structured properties in a continuum framework for reducing the computational demand while employing the appropriated atomic constitutive relations.

Therefore, there is a demand of developing a modeling technique that could analyze the mechanical properties of CNTs at the atomic scale. Considering CNTs as a rolled cylindrical graphite sheet, we step from Young's modulus of the $\mathrm{C}-\mathrm{C}$ bonds counted as Euler beam at atomic scale and extend the theory of classical structural mechanics into the modeling of carbon graphite sheet. The effects of tube curvature on the mechanical properties of SWCNTs are considered in closed-form solutions. The mechanical properties of SWCNTs, including Young's modulus, Poisson's ratio, the length of $\mathrm{C}-\mathrm{C}$ bonds and the angle between the adjacent $\mathrm{C}-\mathrm{C}$ bonds are discussed as functions of nanosized structure.

\section{The Structure of CNTs}

CNTs can be considered as graphite sheets rolled into cylindrical shape. The one-atom-thick graphite sheet looks like chicken wire which is made of a single-carbon-atom thickness. The structure of CNTs as shown in Figure 1 is conveniently explained in terms of the chiral vector integers $(n, m)$ and the chiral angle $\theta[31]$ :

$$
\theta=\tan ^{-1}\left(\frac{\sqrt{3} n}{2 m+n}\right) .
$$

CNTs are classified into three categories named as zigzag $(n, 0): \theta_{z}=0^{\circ}$; armchair $(n, n): \theta_{a}=30^{\circ}$; and chiral $(n$, $m):(m \neq n \neq 0)$. The relationship between radius $r_{\mathrm{cnt}}$ and integers $(n, m)$ is expressed as

$$
r_{\mathrm{cnt}}=\frac{\sqrt{3} L_{\mathrm{CC}}}{2 \pi} \sqrt{n^{2}+m^{2}+m n}
$$

where $L_{\mathrm{CC}}$ is the length of C-C bonds, $0.142 \mathrm{~nm}$, for SWCNTs.

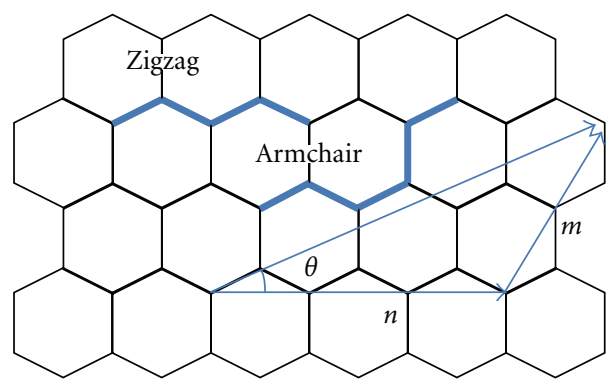

FIGURE 1: Schematic diagram of hexagonal graphite sheet.

\section{Mechanics Model of Graphite Sheet}

From the viewpoint of molecular mechanics, CNTs are treated as a large array of molecules consisting of carbon atoms. According to the Tersoff-Brenner force field theory $[3,32]$, the total molecular potential energy of the force field for nanostructured material can be defined as the sum of bonding and nonbonding energies:

$$
V=\sum V_{r}+\sum V_{\theta}+\sum V_{\varphi}+\sum V_{\omega}+\sum V_{\mathrm{vdw}}+\sum V_{e l},
$$

where $V_{r}$ is the energy induced by bond stretch interaction, $V_{\theta}$ is the energy by the bond angle bending, $V_{\varphi}$ is the energy by the dihedral angle torsion, $V_{\omega}$ is the energy by improper torsion, $V_{\mathrm{vdw}}$ is the energy by the nonbonding van der Waals (vdW) interaction, and $V_{e l}$ is the energy by the electrostatic interaction. As the axial loading, improper torsion and nonbonding interactions subjected to CNTs are very small [33], for covalent systems, the main contributions to the total molecular potential energy come from the first four terms of (3). Simplified system potential energy of CNTs with $\mathrm{C}-\mathrm{C}$ bonds is given as

$$
\begin{aligned}
& V_{r}=\frac{1}{2} k_{r}\left(r-r_{0}\right)^{2}=\frac{1}{2} k_{r}(\Delta r)^{2}, \\
& V_{\theta}=\frac{1}{2} k_{\theta}\left(\theta-\theta_{0}\right)^{2}=\frac{1}{2} k_{\theta}(\Delta \theta)^{2}, \\
& V_{\tau}=V_{\varphi}+V_{\omega}=\frac{1}{2} k_{\tau}(\Delta \phi)^{2},
\end{aligned}
$$

where $k_{r}, k_{\theta}$, and $k_{\tau}$ are the bond stretching, bond bending, and torsional resistance constants, respectively, while $\Delta r$, $\Delta \theta$, and $\Delta \phi$ represent the bond stretching increment, the bond angle change, and the angle change of bond twisting, respectively.

According to classical structural mechanics, the strain energy of a uniform beam in graphite sheet is expressed as

$$
\begin{aligned}
U_{l} & =\frac{E A}{2 L_{\mathrm{CC}}}(\Delta l)^{2}, \\
U_{\theta} & =\frac{E I}{2 L_{\mathrm{CC}}}(\Delta \theta)^{2}, \\
U_{\theta} & =\frac{G J}{2 L_{\mathrm{CC}}}(\Delta \phi)^{2},
\end{aligned}
$$




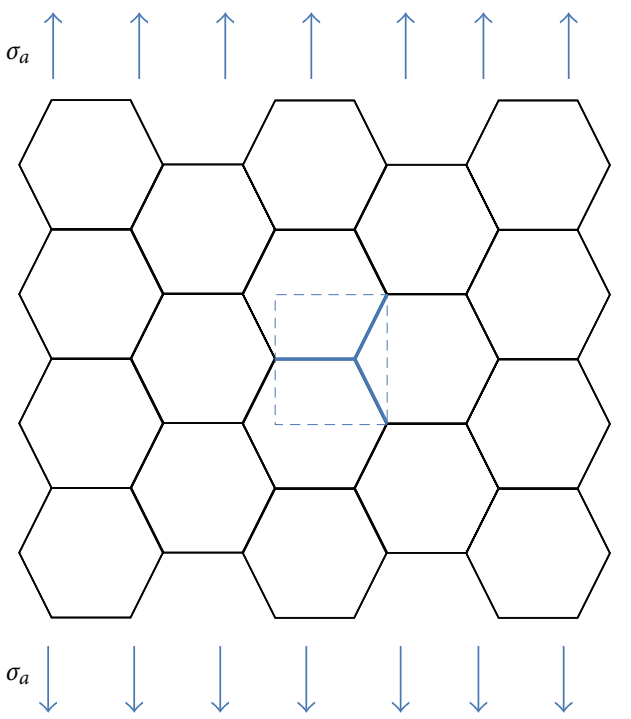

(a)

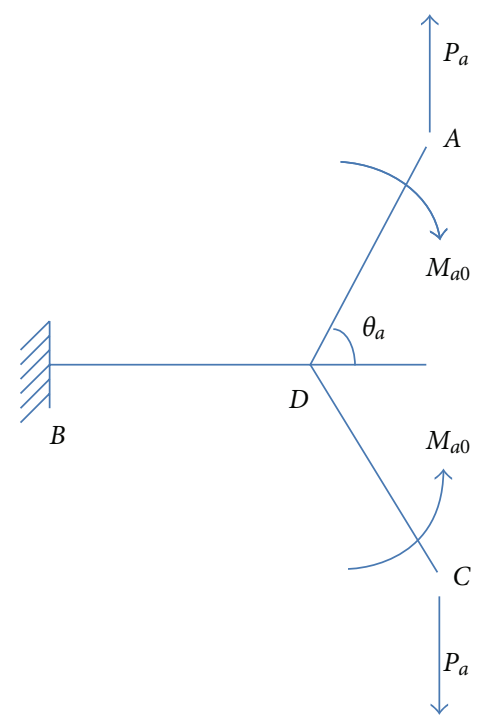

(b)

FIGURE 2: Force analysis of armchair graphite sheet unit subjected to axial tension loading.

where $E A$ is the tensile resistance of beam ( $\mathrm{C}-\mathrm{C}$ bonds), $E I$ is the flexural rigidity, $G J$ is the torsional stiffness, and $\Delta l, \Delta \theta$, and $\Delta \phi$ are the axial stretching deformation, the rotational angle at the end of the beam, and the relative rotation between the ends of the beam, respectively.

Based on energy conservation law, a linkage between the force constants in molecular mechanics and the sectional stiffness parameters in structural mechanics is established. Equations (4) and (5) are equal to each others in one-to-one corresponding directions. The direct relationship between the structural mechanics parameters and the molecular mechanics parameters is deduced as follows:

$$
E A=L_{\mathrm{CC}} k_{r}, \quad E I=L_{\mathrm{CC}} k_{\theta}, \quad G J=L_{\mathrm{CC}} k_{\tau} .
$$

As long as the force constants $k_{r}, k_{\theta}$, and $k_{\tau}$ are known, the sectional stiffness parameters $E A, E I$, and $G J$ can be determined, then the deformation and elastic behavior of CNTs at atomic scale can be modeled. By further analysis of (4) and (5), three more constants of $\mathrm{C}-\mathrm{C}$ bonds $D$ (diameter of $\mathrm{C}-\mathrm{C}$ bonds), $E$, and $G$ depend on $k_{r}, k_{\theta}$, and $k_{\tau}$ :

$$
D=4 \sqrt{\frac{k_{\theta}}{k_{r}}}, \quad E=\frac{k_{r}^{2} L_{\mathrm{CC}}}{4 \pi k_{\theta}}, \quad G=\frac{k_{r}^{2} k_{\tau} L_{\mathrm{CC}}}{8 \pi k_{\theta}^{2}} .
$$

3.1. Young's Modulus for Armchair Graphite Sheet. Knowledge of Young's modulus $(E)$ is the first step towards the material using as a structural element for various applications. SWCNTs can be regarded as a two-dimensional continuum shell which is composed of discrete molecular structures linked by $\mathrm{C}-\mathrm{C}$ bonds. The unrolled graphite sheet of armchair SWCNTs is shown in Figure 2. Figure 2(b) plots the smallest unit of armchair graphite sheet, in which the force $P_{a}$ and moment $M_{a 0}$ are displayed. The unit of armchair graphite sheet can be analyzed based on solid mechanics, and the unit along BD bond is symmetry of both structure and force. Therefore, in terms of the elastic deformation energy, the energy of an armchair unit is written as

$$
W_{a}=\frac{\left(P_{a} \sin \theta_{a}\right)^{2} \times l_{a}}{2 E A}+\int_{0}^{l_{a}} \frac{\left(P_{a} x \cos \theta_{a}-M_{a 0}\right)^{2}}{2 E I} d x,
$$

where $E$ is Young's modulus of beam and $l_{a}$ is the $\mathrm{C}-\mathrm{C}$ bonds of armchair graphite sheet. When the relationship between force $P_{a}$ and moment $M_{a 0}$ is determined, the strain of the unit can be resolved by using energy $W_{a}$.

According to Castigliano's Law, the rotation angel on point $C$ is zero due to symmetry of structure and force that is shown in

$$
\theta_{a c}=\frac{\partial W_{a}}{\partial M_{a 0}}=0
$$

Substituting (8) into (9), the relationship between force $P_{a}$ and moment $M_{a 0}$ is obtained:

$$
M_{a 0}=\frac{P_{a} l_{a}}{2} \cos \theta_{a} .
$$

Meanwhile, the elastic deformation energy $W_{a}$ is rewritten as follows:

$$
W_{a}=\frac{P_{a}^{2} l_{a} \sin ^{2} \theta_{a}}{2 E A}+\frac{P_{a}^{2} l_{a}^{3} \cos ^{2} \theta_{a}}{24 E I} .
$$

Based on Castigliano's law, the displacement of the unit is defined by the elastic deformation energy differential

$$
\delta_{a}=\frac{\partial W_{a}}{\partial P_{a}}=\frac{P_{a} l_{a} \sin ^{2} \theta_{a}}{E A}+\frac{P_{a} l_{a}^{3} \cos ^{2} \theta_{a}}{12 E I},
$$

and the strain of armchair graphite sheet is defined as follows:

$$
\varepsilon_{a}=\frac{\delta_{a}}{l_{a} \sin \theta_{a}} .
$$




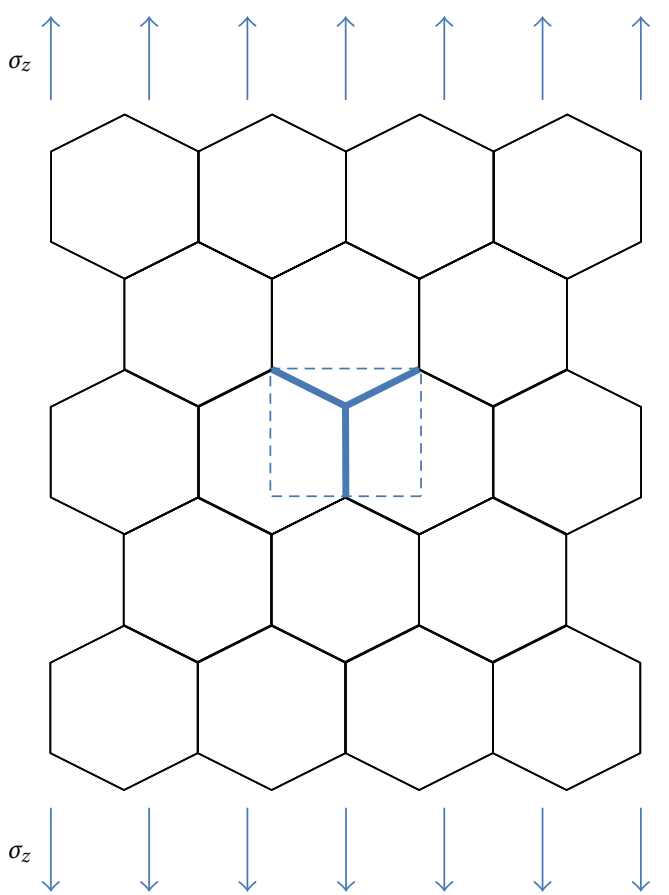

(a)

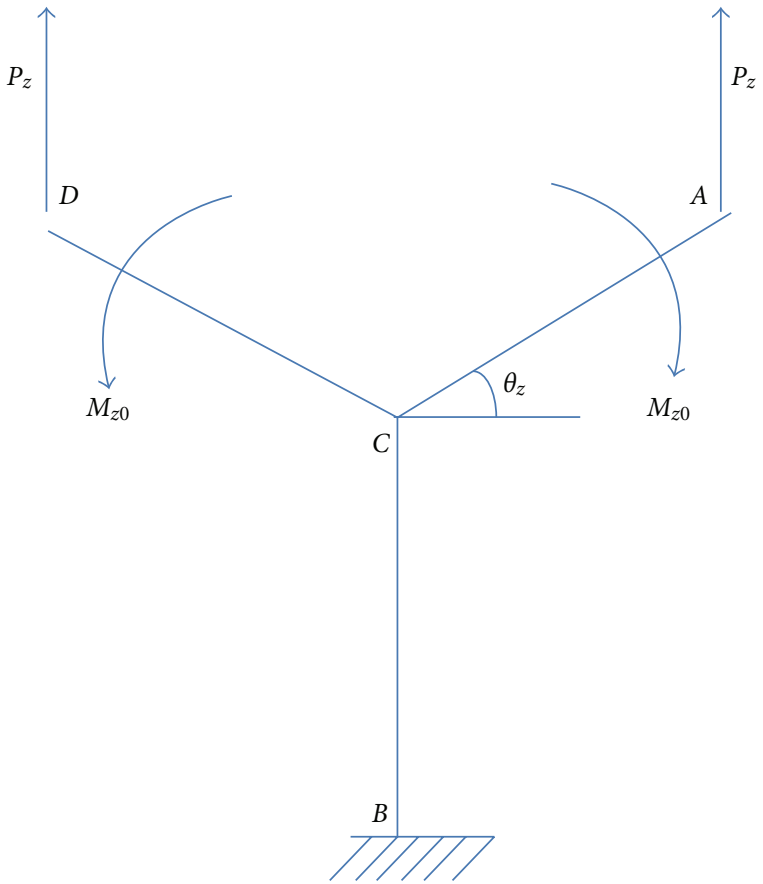

(b)

FIGURE 3: Force analysis of zigzag graphite sheet unit subjected to axial tension loading.

For an armchair graphite sheet being subjected to the tension stress $\sigma_{a}$, the equation of static equilibrium of the unit is expressed as

$$
\sigma_{a} t l_{a}\left(1+\cos \theta_{a}\right)=P_{a} .
$$

Based on Hooke's law, the relationship of the tension stress and the strain is

$$
\sigma_{a}=E_{a} \varepsilon_{a} .
$$

When $\theta_{a}=60^{\circ}$, Young's modulus of armchair graphite sheet $E_{a}$ yields

$$
E_{a}=\frac{\sqrt{3} E}{t}\left(\frac{9 l_{a}}{4 A}+\frac{l_{a}^{3}}{16 I}\right)^{-1}
$$

where $t$ is the thickness of the graphite sheet.

3.2. Young's Modulus for the Zigzag Graphite Sheet. For a zigzag graphite sheet, the analytical approach is similar to that of the armchair graphite sheet. The unit of a zigzag graphite sheet is drawn in Figure 3, and the energy of the unit is drawn in

$$
W_{z}=\frac{P_{z}^{2} l_{z}}{E A}\left(\sin ^{2} \theta_{z}+2\right)+\frac{P_{z}^{2} l_{z}^{3} \cos ^{2} \theta_{z}}{12 E I},
$$

where $l_{a}$ is the $\mathrm{C}-\mathrm{C}$ bonds of the zigzag graphite sheet. The displacement of the zigzag unit is defined as

$$
\delta_{z}=\frac{P_{z} l_{z}}{E A}\left(\sin ^{2} \theta_{z}+2\right)+\frac{P_{z} l_{z}^{3} \cos ^{2} \theta_{z}}{12 E I} .
$$

Then the strain of per unit length is obtained as follows:

$$
\varepsilon_{z}=\frac{\delta_{z}}{l_{z}\left(\sin \theta_{z}+1\right)} .
$$

For a zigzag graphite sheet being subjected to the tension stress $\sigma_{z}$, the force acting on the unit can be written as

$$
\sigma_{z} t d \cos \theta_{z}=P_{z}
$$

When $\theta_{z}=30^{\circ}$, we can obtain Young's modulus $E_{z}$ of zigzag graphite sheet:

$$
E_{z}=\frac{\sqrt{3} E}{t}\left(\frac{9 l_{z}}{4 A}+\frac{l_{z}^{3}}{16 I}\right)^{-1}
$$

\section{Molecular Mechanics Model of SWCNTs}

SWCNTs can be ideally constructed starting from a graphite sheet. According to the chiral vector, there are three kinds of structure, as shown in Figure 4. After the two-dimensional sheets are rolled into a three-dimensional tube, intrinsic properties of the structure will be changed, such as mechanical and physical characteristics.

4.1. Angle Measurement for SWCNTs. Figure 4(a) shows an armchair SWCNT. According to (2) the radius of armchair SWCNTs is $r_{a \mathrm{cnt}}=3 n \cdot L_{\mathrm{CC}} / 2 \pi$. Meanwhile, by geometric 

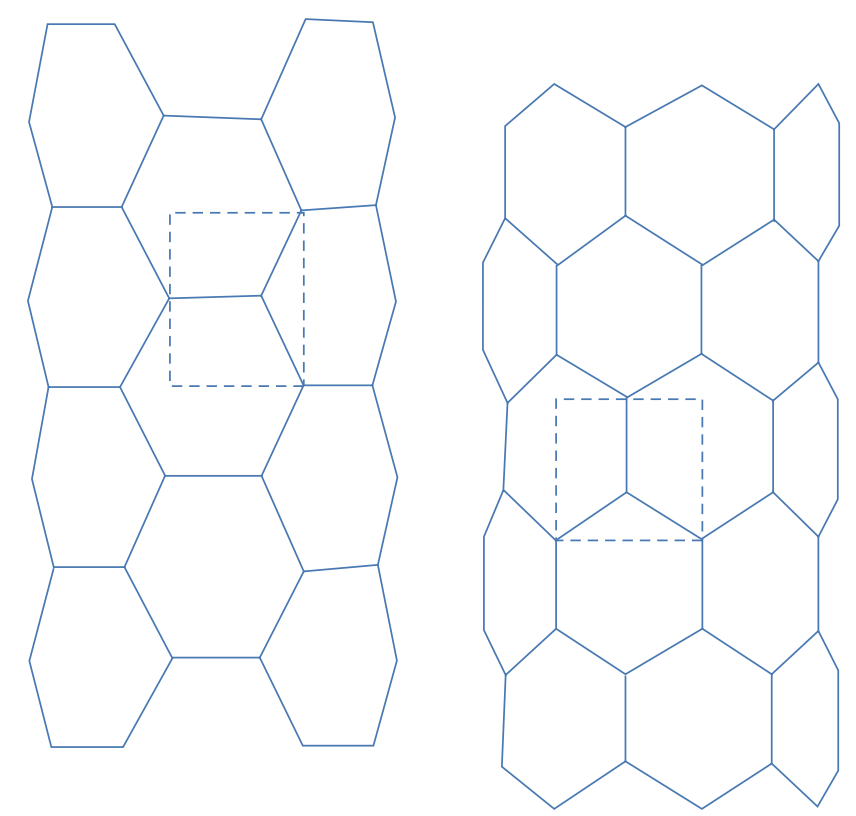

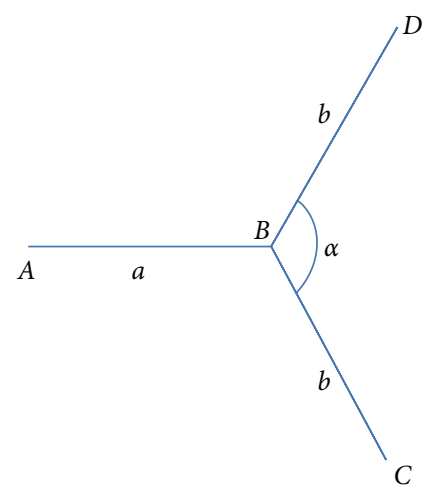

(a)

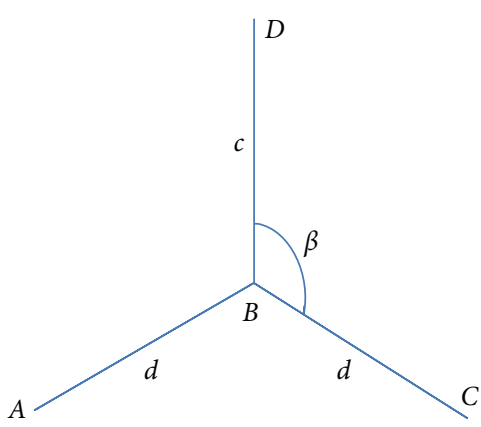

(b)

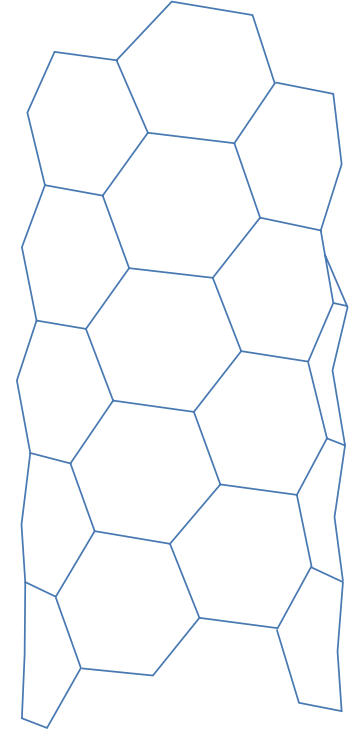

(c)

FIgURE 4: Classification of SWCNTs by chiral vector and chiral angle (a) armchair SWCNT, (b) zigzag SWCNT, and (c) chiral SWCNT.

relationships in cylindrical coordinates shown in Figure 4(a), we obtain the coordinates of carbon atoms:

$$
\begin{aligned}
& A=\left(r_{a c \mathrm{nt}}, 0,0\right), \\
& B=\left(r_{a \mathrm{cnt}} \cos \frac{2 \pi}{3 n}, r_{a \mathrm{cnt}} \sin \frac{2 \pi}{3 n}, 0\right), \\
& C=\left(r_{a \mathrm{cnt}} \cos \frac{\pi}{n}, r_{a \mathrm{cnt}} \sin \frac{\pi}{n},-\frac{\sqrt{3}}{2} L_{\mathrm{CC}}\right), \\
& D=\left(r_{a \mathrm{cnt}} \cos \frac{\pi}{n}, r_{a \mathrm{cnt}} \sin \frac{\pi}{n}, \frac{\sqrt{3}}{2} L_{\mathrm{CC}}\right) .
\end{aligned}
$$

Because of the effect of curvature, the lengths of the bonds between carbon atoms in vector space are shorter than those in plane and characterized in different numerical values, which are displayed in Figure 4(a) and given as follows:

$$
\begin{aligned}
& a=|\overrightarrow{B A}|=\frac{3 n}{2 \pi} \sqrt{2-2 \cos \frac{2 \pi}{3 n}} L_{\mathrm{CC}}, \\
& b=|\overrightarrow{B D}|=|\overrightarrow{B C}|=\frac{3 n}{2 \pi} \sqrt{2-2 \cos \frac{\pi}{3 n}+\frac{\pi^{2}}{3 n^{2}}} L_{\mathrm{CC}} .
\end{aligned}
$$

In cylindrical coordinates, on account of the curvature, the included angles between the adjacent bonds in a carbon cycle of SWCNTs are different from those of graphite sheets. In accordance with geometrical relationships, the included angle between the adjacent bonds for an arbitrary unit of armchair SWCNTs plotted in Figure 4(a) relates with coordinate figures of carbon atoms in the unit:

$$
\cos \alpha=\frac{\overrightarrow{B D} \cdot \overrightarrow{B C}}{|\overrightarrow{B D}||\overrightarrow{B C}|} .
$$


Substituting (23) into (24), we obtain the included angle of armchair SWCNTs

$$
\alpha=\cos ^{-1}\left(\frac{6 n^{2}(1-\cos \pi / 3 n)-\pi^{2}}{6 n^{2}(1-\cos \pi / 3 n)+\pi^{2}}\right)
$$

Figure 4(b) shows a zigzag SWCNT. According to (2) the radius of zigzag SWCNTs is $r_{z \mathrm{cnt}}=\sqrt{3} n \cdot L_{\mathrm{CC}} / 2 \pi$. Considering geometric relationships in cylindrical coordinates, the coordinate figures are described as

$$
\begin{aligned}
A & =\left(r_{z \mathrm{cnt}}, 0,0\right), \\
B & =\left(r_{z \mathrm{cnt}} \cos \frac{\pi}{n}, r_{z \mathrm{cnt}} \sin \frac{\pi}{n}, \frac{1}{2} L_{\mathrm{CC}}\right), \\
C & =\left(r_{z \mathrm{cnt}} \cos \frac{2 \pi}{n}, r_{z \mathrm{cnt}} \sin \frac{2 \pi}{n}, 0\right), \\
D & =\left(r_{z \mathrm{cnt}} \cos \frac{\pi}{n}, r_{z \mathrm{cnt}} \sin \frac{\pi}{n}, \frac{3}{2} L_{\mathrm{CC}}\right) .
\end{aligned}
$$

The distances of the bonds in vector space are as follows:

$$
\begin{aligned}
& c=|\overrightarrow{B D}|=L_{\mathrm{CC}} \\
& d=|\overrightarrow{B A}|=|\overrightarrow{B C}|=\frac{\sqrt{3} n}{2 \pi} \sqrt{2-2 \cos \frac{\pi}{n}+\frac{\pi^{2}}{3 n^{2}}} L_{\mathrm{CC}} .
\end{aligned}
$$

For zigzag SWCNTs, the analysis method resembles armchair SWCNTs; we calculate the included angle for zigzag SWCNTs as follows:

$$
\beta=\cos ^{-1}\left(\frac{-\pi}{\sqrt{6 n^{2}(1-\cos \pi / n)+\pi^{2}}}\right) .
$$

4.2. Elastic Properties for SWCNTs. Considering space curvature, Young's modulus of SWCNTs in cylindrical coordinates depends on bond length and included angle between two bonds. The method for calculating Young's modulus of armchair SWCNTs in three-dimensions resembles that in two dimensions. Affecting factors in three dimensional coordinates of Young's modulus of armchair SWCNTs are mentioned in (23) and (25); Young's modulus of armchair SWCNTs is expressed in

$$
E_{a \mathrm{cnt}}=\xi \lambda_{a}\left(k_{a 1} \frac{1}{A}+k_{a 2} \frac{1}{12 I}\right)^{-1}
$$

where $\xi=(E / t), \lambda_{a}=\sin (\alpha / 2) / a+b \cos (\alpha / 2), k_{a 1}=\sin ^{2} \alpha / 2$, and $k_{a 2}=b^{2} \cos ^{2} \alpha / 2$.

We obtain Young's modulus of zigzag SWCNTs given in the following in the same way

$$
E_{z \mathrm{cnt}}=\xi \lambda_{z}\left(k_{z 1} \frac{1}{A}+k_{z 2} \frac{1}{12 I}\right)^{-1}
$$

where $\lambda_{z}=(d \sin (\beta-(\pi / 2))+c) /(d \cos (\beta-(\pi / 2))), k_{z 1}=$ $d \sin ^{2}(\beta-(\pi / 2))+2 c$, and $k_{z 2}=\cos ^{2}(\beta-(\pi / 2)) d^{3}$.
4.3. Poisson's Ratio for SWCNTs. For armchair SWCNTs, the stretch deformations of the bonds caused by concentrated force and bending moment are schematically signed in Figure 5(a) to analyze Poisson's ratio. The equilibrium of force $P_{a}$ and bending moment $M_{a}$ can be computed from Figure 5(a)

$$
2 M_{a}=P_{a} b \sin \frac{\alpha}{2}
$$

According to geometric properties and elastic theory, taking the included angle between two bonds in three dimensions into consideration, we obtain the equilibrium equations about extension variation of the $\mathrm{C}-\mathrm{C}$ bonds

$$
\begin{aligned}
& \delta_{a}^{P 1}=\frac{P_{a} \sin \alpha / 2}{E A}, \\
& \delta_{a}^{P 2}=\frac{P_{a} b^{3} \cos \alpha / 2}{3 E I}, \\
& \delta_{a}^{M}=-\frac{M_{a} b^{2} \cos \alpha / 2}{2 E I},
\end{aligned}
$$

where $E A$ and $E I$ are the tensile resistance and the flexural rigidity of beam, and $\delta_{a}^{P 1}, \delta_{a}^{P 2}$, and $\delta_{a}^{M}$ are the deformations of beam caused by force $P_{a}$ and bending moment $M_{a}$, respectively. The strains in axial direction and circumferential direction are defined as follows, respectively:

$$
\begin{aligned}
& \varepsilon_{a 1}=\frac{\delta_{a}^{P 1} \cos (\alpha / 2)-\left(\delta_{a}^{P 2}+\delta_{a}^{M}\right) \sin \alpha / 2}{a+b \cos \alpha / 2}, \\
& \varepsilon_{a 2}=\frac{\delta_{a}^{P 1} \sin (\alpha / 2)+\left(\delta_{a}^{P 2}+\delta_{a}^{M}\right) \cos \alpha / 2}{b \sin \alpha / 2} .
\end{aligned}
$$

Poisson's ratio of armchair SWCNTs can be defined as the ratio between circumferential strain, and axial strain, substituting (31) and (32) into (33) we can get Poisson's ratio of armchair SWCNTs

$\nu_{a}=\frac{\varepsilon_{a 1}}{\varepsilon_{a 2}}=-\frac{\left(12 I-A b^{3}\right) \sin ^{2}(\alpha / 2) \cos (\alpha / 2)}{\left(12 I \sin ^{2}(\alpha / 2)+A b^{3} \cos ^{2} \alpha / 2\right)((a / b)+\cos \alpha / 2)}$

For zigzag SWCNTs, the analysis step is similar to that for armchair SWCNTs. To analyze Poisson's ratio of zigzag SWCNTs, Figure 5(b) schematically signs the stretch deformations of the bonds caused by force $P_{z}$ and bending moment $M_{z}$; the relationship of force and bending moment is given in

$$
2 M_{z}=P_{z} b \sin \frac{\alpha}{2}
$$




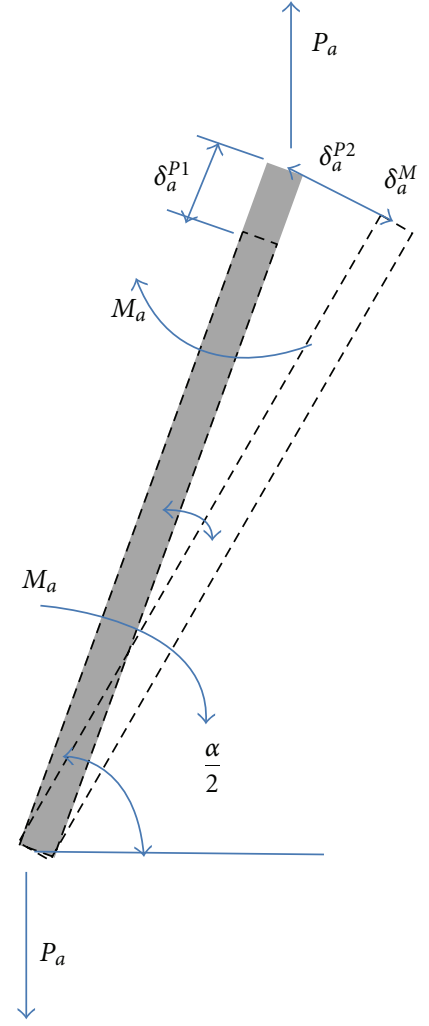

(a)

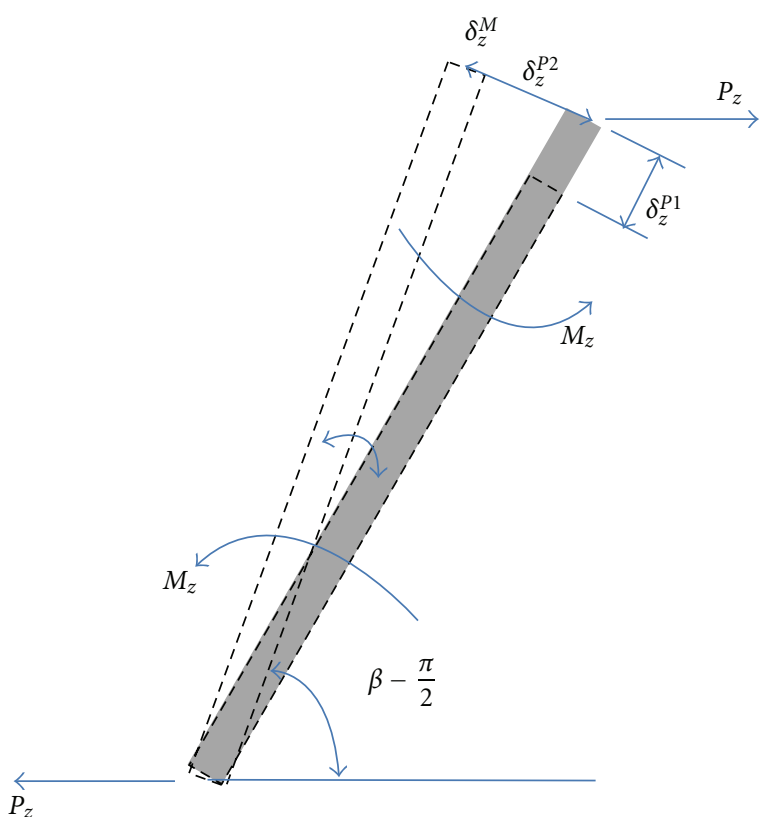

(b)

FIgURE 5: Analysis of axial deformation and angular displacement of armchair and zigzag SWCNTs.

The equilibrium equations about extension variation of the bond are described as

$$
\begin{aligned}
& \delta_{z}^{P 1}=\frac{P_{z} \sin (\beta-(\pi / 2))}{E A}, \\
& \delta_{z}^{P 2}=\frac{P_{z} d^{3} \cos (\beta-(\pi / 2))}{3 E I}, \\
& \delta_{z}^{P 3}=\frac{2 P_{z} \sin (\beta-(\pi / 2))}{E A}, \\
& \delta_{z}^{M}=-\frac{M_{z} d^{2} \cos (\beta-(\pi / 2))}{2 E I} .
\end{aligned}
$$

The strains in axial direction and circumferential direction are defined as follows, respectively:

$$
\begin{aligned}
& \mathcal{E}_{z 1}=\frac{\delta_{z}^{P 1} \cos (\beta-(\pi / 2))-\left(\delta_{z}^{P 2}+\delta_{z}^{M}\right) \sin (\beta-(\pi / 2))}{d \cos (\pi-\beta)}, \\
& \varepsilon_{z 2}=\frac{\delta_{z}^{P 1} \sin (\beta-(\pi / 2))+\left(\delta_{z}^{P 2}+\delta_{z}^{M}\right) \cos (\beta-(\pi / 2))+\delta_{z}^{P 3}}{c+d \sin (\pi-\beta)} .
\end{aligned}
$$

Poisson's ratio of armchair SWCNTs can be defined as the ratio between circumferential strain and axial strain; then we obtain

$$
\begin{aligned}
v_{z} & =\frac{\varepsilon_{z 1}}{\mathcal{E}_{z 2}} \\
& =\frac{\left(12 I-A b^{3}\right)((c / d)+\sin (\beta-(\pi / 2))) \sin (\beta-(\pi / 2))}{\left(12 I \sin ^{2}(\beta-(\pi / 2))+A d^{3} \cos ^{2}(\beta-(\pi / 2))+24 A\right)} .
\end{aligned}
$$

\section{Results and Discussions}

The atomic-based continuum mechanic approach described in the previous section was implemented for studying the effective elastic properties of graphite sheets and SWCNTs. In this section, the mechanical characteristics of graphite sheet and SWCNTs are examined.

5.1. Mechanics Model of Graphite Sheet. In the present simulation, $k_{r} / 2=46900 \mathrm{kcal} / \mathrm{mole} / \mathrm{nm}^{2}, k_{\theta} / 2=63 \mathrm{kcal} /$ mole $/ \mathrm{rad}^{2}$ and $k_{\tau} / 2=20 \mathrm{kcal} / \mathrm{mole} / \mathrm{rad}^{2}$, are taken [34]. $L_{\mathrm{CC}}$ and $t$ are $0.142 \mathrm{~nm}$ and $0.34 \mathrm{~nm}$, respectively. According to (6) and (7), the constants of beam are obtained and summarized in Table 1. Comparing the evaluated elastic moduli of graphite sheets with the literature results, Tserpes [8] and Kalamkarov [9] reported $E=5.49 \mathrm{TPa}$ and 
TABLE 1: Geometrical and material properties of $\mathrm{C}-\mathrm{C}$ bonds.

\begin{tabular}{lccc}
\hline Beam element & Abbreviation & Value & \\
\hline Diameter & $D$ & 0.146 & Unit \\
Cross-section area & $A$ & $1.678 \times 10^{-2}$ & $\mathrm{~nm}$ \\
Moment of inertia & $I$ & $2.241 \times 10^{-5}$ & $\mathrm{~nm}^{2}$ \\
Young's modulus & $E$ & 5.530 & $\mathrm{~nm}^{4}$ \\
Shear elastic modulus & $G$ & 0.871 & $\mathrm{TPa}$ \\
\hline
\end{tabular}

$G=0.871 \mathrm{TPa}, E=5.488 \mathrm{TPa}$ and $G=0.8711 \mathrm{TPa}$ of $\mathrm{C}-\mathrm{C}$ bonds, respectively.

Comparing (16) to (21), Young's moduli of the armchair graphite sheet and the zigzag graphite sheet have the same expression form except for $l_{a}$ and $l_{z}$. Because the carbon cycle is a regular hexagon, graphite sheets have $l_{a}=l_{z}=L_{C C}$. We obtain Young's moduli of both armchair and zigzag graphite sheets as $1.0424 \mathrm{TPa}$, which is close to the value of $1.033 \mathrm{TPa}$ calculated by $\mathrm{Li}$ and Chou [31] and the value of $1.04 \mathrm{TPa}$ computed by Shokrieh and Rafiee [11]. It can be seen that the predicted values obtained from the model at the atomic scale agree well with those reported in the literatures.

\subsection{Molecular Mechanics Model of SWCNTs. Since SWCNTs} are defined as rolled graphite sheets, the lengths of bonds in vector space are changed owing to the effect of curvature. For armchair SWCNTs rolled by armchair graphite sheets, all of the lengths of $\mathrm{C}-\mathrm{C}$ bonds become shorter because of the connection with curvature in circumferential direction, while, for zigzag SWCNTs rolled by zigzag graphite sheet, the length $c$ is only in vertical direction equal to the $\mathrm{C}-\mathrm{C}$ bonds length of graphite sheets. According to (23) and (27), the variation of $\mathrm{C}-\mathrm{C}$ bonds lengths with radius are shown in Figure 6. With increasing radius of SWCNTs, the bond lengths $a, b$, and $d$ approach to the length $c=0.142 \mathrm{~nm}$ which is the bond length of graphite itself, owing to the curvature approaching to zero in infinite radius.

When graphite sheets are rolled into SWCNTs, the lengths and spatial relations of $\mathrm{C}-\mathrm{C}$ bonds change obviously. Figure 7 shows the variation of included angles of two adjacent $\mathrm{C}-\mathrm{C}$ bonds with radius according to (25) and (28). It expresses that the included angles are quite sensitive to SWCNTs in small radius. The included angle of zigzag SWCNTs is a little larger than that of armchair SWCNTs in the same radius. As being larger than $1.1 \mathrm{~nm}$, the radius makes less influence on the included angles of both armchair and zigzag SWCNTs, and the two lines show a tendency to be 120 degree which is close to that of graphite sheets, due to the SWCNTs being regarded as graphite sheet when radius is infinite.

Comparing (29) to (30), Young's moduli of armchair and zigzag SWCNTs have the same expression form except for coefficients $\lambda_{a}, k_{a 1}, k_{a 2}$ and $\lambda_{z}, k_{z 1}, k_{z 2}$. As the two Young's moduli shown in Figure 8, Young's moduli predicted by the present theory decrease monotonically with the increase of radius. Young's modulus of armchair SWCNTs is slightly higher than that of zigzag SWCNTs with the same smaller

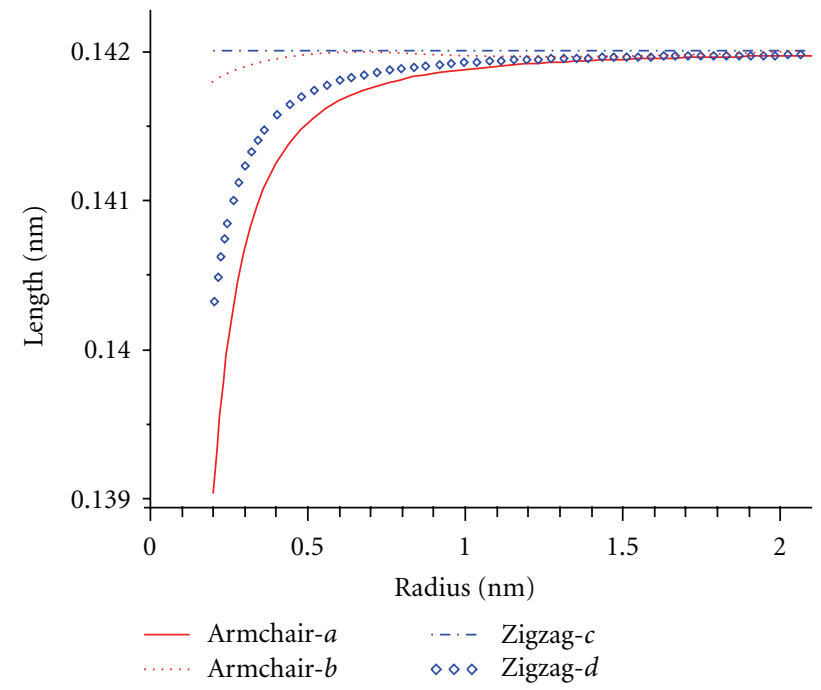

FIgURE 6: $\mathrm{C}-\mathrm{C}$ bonds lengths of armchair SWCNTs and zigzag SWCNTs.

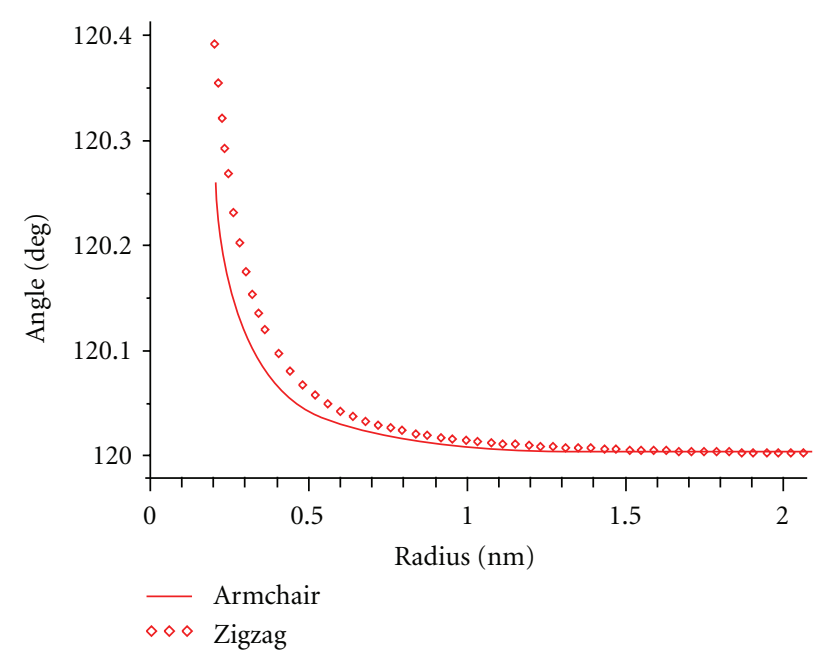

Figure 7: Included angles of adjacent $\mathrm{C}-\mathrm{C}$ bonds in armchair SWCNTs and zigzag SWCNTs.

radius. When the radius is larger, the two declining curves gradually develop into horizontal lines, which tend to be a constant of $1.0424 \mathrm{TPa}$. The value is Young's modulus of graphite sheet. 


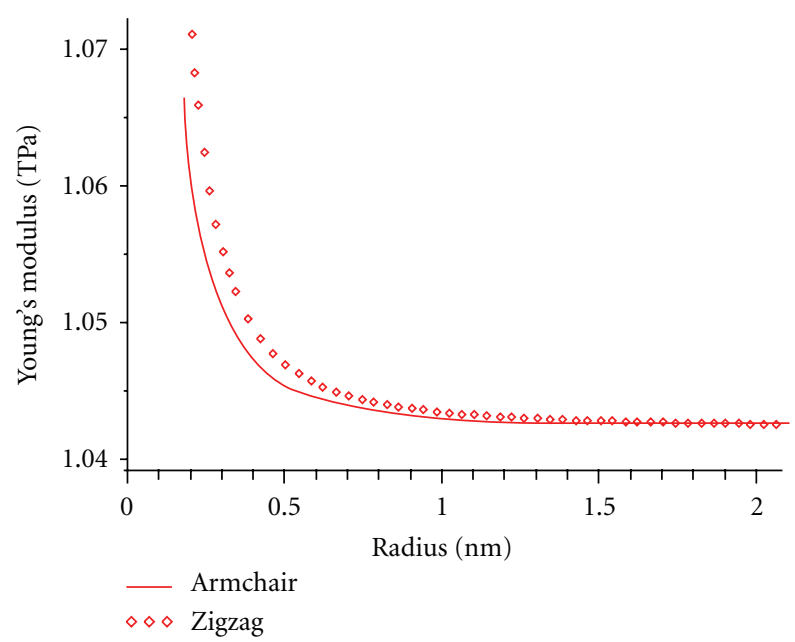

FIGURE 8: Young's modulus of armchair SWCNTs and zigzag SWCNTs.

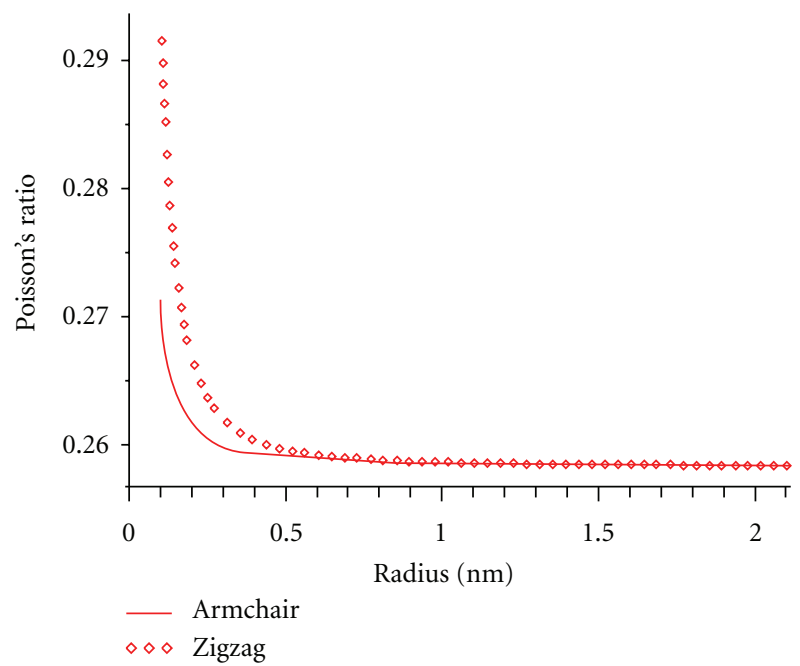

FIGURE 9: Poisson's ratio of armchair SWCNTs and zigzag SWCNTs.

Considering the variations of length and included angles of two adjacent $\mathrm{C}-\mathrm{C}$ bonds, from graphite sheets into SWCNTs, Figure 9 shows the variations of Poisson's ratio of the armchair and zigzag SWCNTs as a function of nanotube radius. It is found that, in general, Poisson's ratio of armchair SWCNTs is slightly higher than that of zigzag SWCNTs. Poisson's ratios of armchair and zigzag SWCNTs show a smoothly monotonic decreasing versus increasing radius. When the radius of SWCNTs is larger, the two curves tend to be a straight line which means that Poisson's ratios approach to be a steady value of 0.26 , which makes a great agreement with those reported in the literatures $[6,33]$.

\section{Conclusions}

The mechanical properties of both armchair and zigzag SWCNTs are characterized by using continuum mechanics in the atomic scale. In terms of the conjunction of TersoffBrenner force field method and energy conservation law, the graphite sheet is of isotropic property and Young's modulus of graphite sheet is obtained to be $1.04 \mathrm{TPa}$. Furthermore, considering the variations of the length and the included angle of two adjacent $\mathrm{C}-\mathrm{C}$ bonds rolled from graphite sheets into SWCNTs, Young's modulus and Poisson's ratio of SWNCTs with armchair and zigzag structures are investigated as a function of SWCNT radius. We predict that Young's modulus and Poisson's ratio of SWNCTs are influenced obviously by relatively smaller radius while being little affected by larger radius. We are confident that this model provides a useful method to analyze mechanical properties of CNTs and other nanosized structures at the atomic scale.

\section{Acknowledgment}

This work was supported by Grant-in-Aid for Global COE Program by the Ministry of Education, Culture, Sports, Science and Technology.

\section{References}

[1] S. Iijima, "Helical microtubules of graphitic carbon," Nature, vol. 354, no. 6348, pp. 56-58, 1991.

[2] F. Li, H. M. Cheng, S. Bai, G. Su, and M. S. Dresselhaus, "Tensile strength of single-walled carbon nanotubes directly measured from their macroscopic ropes," Applied Physics Letters, vol. 77, no. 20, pp. 3161-3163, 2000.

[3] W. H. Chen, H. C. Cheng, and Y. L. Liu, "Radial mechanical properties of single-walled carbon nanotubes using modified molecular structure mechanics," Computational Materials Science, vol. 47, pp. 985-993, 2010.

[4] Z. Spitalskya, D. Tasisb, K. Papagelisb, and C. Galiotis, "Carbon nanotube-polymer composites: chemistry, processing, mechanical and electrical properties," Progress in Polymer Science, vol. 35, pp. 357-401, 2010.

[5] M. A. L. Manchado, L. Valentini, J. Biagiotti, and J. M. Kenny, "Thermal and mechanical properties of single-walled carbon nanotubes-polypropylene composites prepared by melt processing," Carbon, vol. 43, no. 7, pp. 1499-1505, 2005.

[6] T. Natsuki, K. Tantrakarn, and M. Endo, "Effects of carbon nanotube structures on mechanical properties," Applied Physics A, vol. 79, no. 1, pp. 117-124, 2004.

[7] M. R. Falvo, G. J. Clary, R. M. Taylor II et al., "Bending and buckling of carbon nanotubes under large strain," Nature, vol. 389, no. 6651, pp. 582-584, 1997.

[8] K. I. Tserpes and P. Papanikos, "Finite element modeling of single-walled carbon nanotubes," Composites Part B, vol. 36, no. 5, pp. 468-477, 2005.

[9] A. L. Kalamkarov, A. V. Georgiades, S. K. Rokkam, V. P. Veedu, and M. N. Ghasemi-Nejhad, "Analytical and numerical techniques to predict carbon nanotubes properties," International Journal of Solids and Structures, vol. 43, no. 22-23, pp. 68326854, 2006.

[10] O. L. Blakslee, D. G. Proctor, E. J. Seldin, G. B. Spence, and T. Weng, "Elastic constants of compression-annealed pyrolytic graphite," Journal of Applied Physics, vol. 41, no. 8, pp. 33733382, 1970. 
[11] M. M. Shokrieh and R. Rafiee, "Prediction of Young's modulus of graphene sheets and carbon nanotubes using nanoscale continuum mechanics approach," Materials and Design, vol. 31, no. 2, pp. 790-795, 2010.

[12] K. N. Kudin, G. E. Scuseria, and B. I. Yakobson, " $\mathrm{C}_{2} \mathrm{~F}$, BN, and C nanoshell elasticity from $a b$ initio computations," Physical Review B, vol. 64, no. 23, Article ID 235406, 10 pages, 2001.

[13] O. Lourie and H. D. Wagner, "Evaluation of Young's modulus of carbon nanotubes by micro-Raman spectroscopy," Journal of Materials Research, vol. 13, no. 9, pp. 2418-2422, 1998.

[14] M. M. J. Treacy, T. W. Ebbesen, and J. M. Gibson, "Exceptionally high Young's modulus observed for individual carbon nanotubes," Nature, vol. 381, no. 6584, pp. 678-680, 1996.

[15] J.-P. Salvetat, J.-M. Bonard, N. B. Thomson et al., "Mechanical properties of carbon nanotubes," Applied Physics A, vol. 69, no. 3, pp. 255-260, 1999.

[16] N. Yao and V. Lordi, "Young's modulus of single-walled carbon nanotubes," Journal of Applied Physics, vol. 84, no. 4, pp. 1939 1943, 1998.

[17] P. M. Ajayan, O. Stephan, C. Colliex, and D. Trauth, "Aligned carbon nanotube arrays formed by cutting a polymer resinnanotube composite," Science, vol. 265, no. 5176, pp. 1212 1214, 1994.

[18] P. Poncharal, Z. L. Wang, D. Ugarte, and W. A. de Heer, "Electrostatic deflections and electromechanical resonances of carbon nanotubes," Science, vol. 283, no. 5407, pp. 1513-1516, 1999.

[19] M. R. Falvo, G. J. Clary, R. M. Taylor II et al., "Bending and buckling of carbon nanotubes under large strain," Nature, vol. 389, no. 6651, pp. 582-584, 1997.

[20] E. W. Wong, P. E. Sheehan, and C. M. Lieber, "Nanobeam mechanics: elasticity, strength, and toughness of nanorods and nanotubes," Science, vol. 277, no. 5334, pp. 1971-1975, 1997.

[21] J.-P. Salvetat, A. J. Kulik, J.-M. Bonard et al., "Elastic modulus of ordered and disordered multiwalled carbon nanotubes," Advanced Materials, vol. 11, no. 2, pp. 161-165, 1999.

[22] F. Ding, "Theoretical study of the stability of defects in singlewalled carbon nanotubes as a function of their distance from the nanotube end," Physical Review B, vol. 72, Article ID 245409, 7 pages, 2005.

[23] K. M. Liew, C. H. Wong, X. Q. He, M. J. Tan, and S. A. Meguid, "Nanomechanics of single and multiwalled carbon nanotubes," Physical Review B, vol. 69, no. 11, Article ID 115429, 8 pages, 2004.

[24] V. U. Unnikrishnan, D. Banerjee, and J. N. Reddy, "Atomisticmesoscale interfacial resistance based thermal analysis of carbon nanotube systems," International Journal of Thermal Sciences, vol. 47, no. 12, pp. 1602-1609, 2008.

[25] Y. Zhou, M. A. Baseer, H. Mahfuz, and S. Jeelani, "Monte Carlo simulation on tensile failure process of unidirectional carbon fiber reinforced nano-phased epoxy," Materials Science and Engineering A, vol. 420, no. 1-2, pp. 63-71, 2006.

[26] G. Overney, W. Zhong, and D. Tománek, "Structural rigidity and low frequency vibrational modes of long carbon tubules," Zeitschrift für Physik D, vol. 27, no. 1, pp. 93-96, 1993.

[27] J. P. Lu, "Elastic properties of single and multilayered nanotubes," Journal of Physics and Chemistry of Solids, vol. 58, no. 11, pp. 1649-1652, 1997.

[28] B. I. Yakobson, C. J. Brabec, and J. Bernholc, "Nanomechanics of carbon tubes: instabilities beyond linear response," Physical Review Letters, vol. 76, pp. 2511-2514, 1996.

[29] J. P. Lu, "Elastic properties of carbon nanotubes and nanoropes," Physical Review Letters, vol. 79, no. 7, pp. 1297 1300, 1997.
[30] S. A. Meguid, J. M. Wernik, and Z. Q. Cheng, "Atomisticbased continuum representation of the effective properties of nano-reinforced epoxies," The International Journal of Solids and Structures, vol. 47, pp. 1723-1736, 2010.

[31] C. Li and T.-W. Chou, "A structural mechanics approach for the analysis of carbon nanotubes," The International Journal of Solids and Structures, vol. 40, no. 10, pp. 2487-2499, 2003.

[32] J.-L. Tsai, S.-H. Tzeng, and Y.-T. Chiu, "Characterizing elastic properties of carbon nanotubes/polyimide nanocomposites using multi-scale simulation," Composites Part B, vol. 41, pp. 106-115, 2009.

[33] T. Natsuki, K. Tantrakarn, and M. Endo, "Prediction of elastic properties for single-walled carbon nanotubes," Carbon, vol. 42, no. 1, pp. 39-45, 2004.

[34] W. D. Cornell, P. Cieplak, C. I. Bayly et al., "A second generation force field for the simulation of proteins, nucleic acids, and organic molecules," Journal of the American Chemical Society, vol. 117, no. 19, pp. 5179-5197, 1995. 

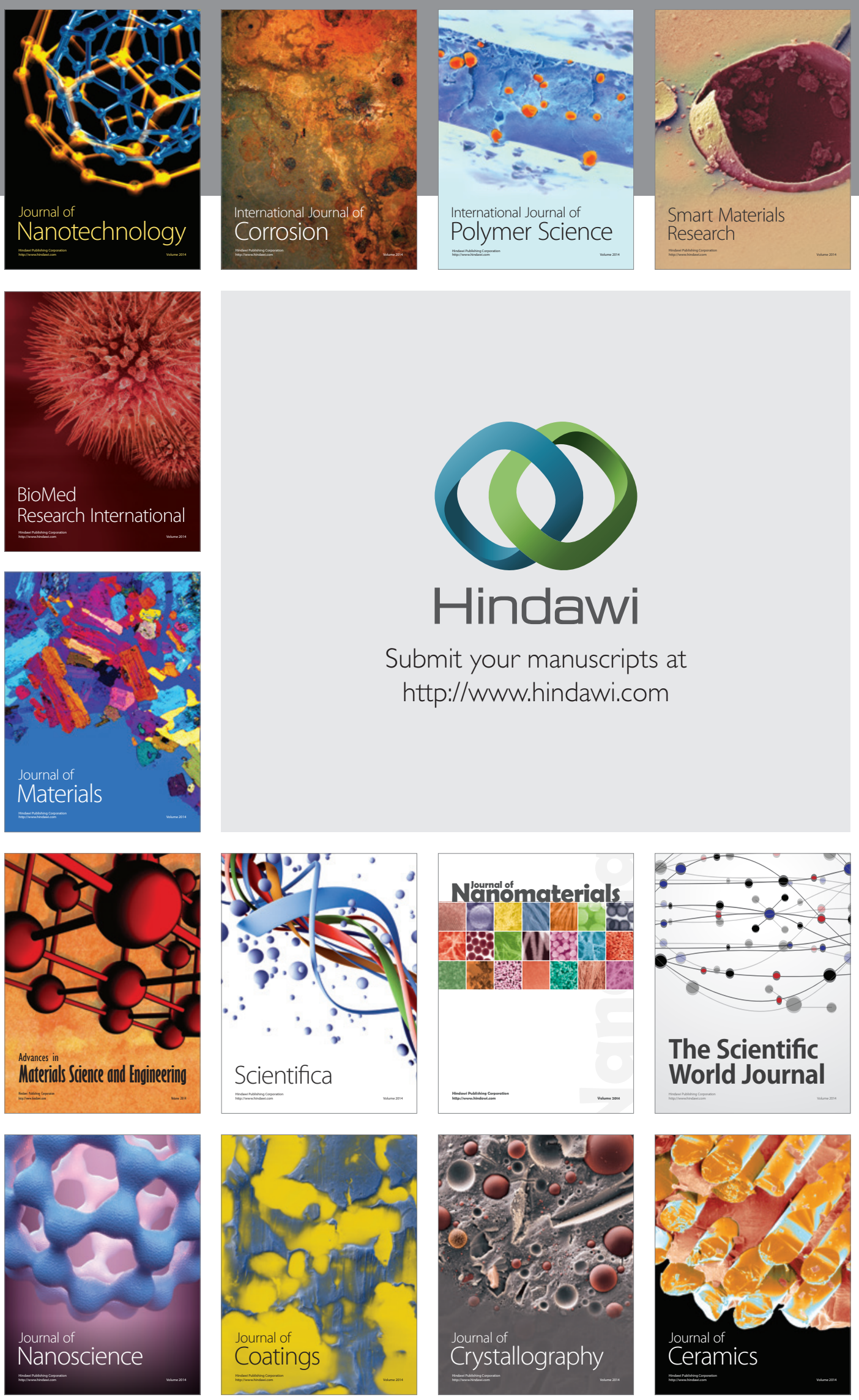

The Scientific World Journal

Submit your manuscripts at

http://www.hindawi.com

\section{World Journal}

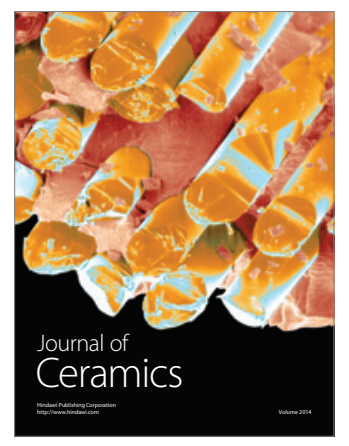

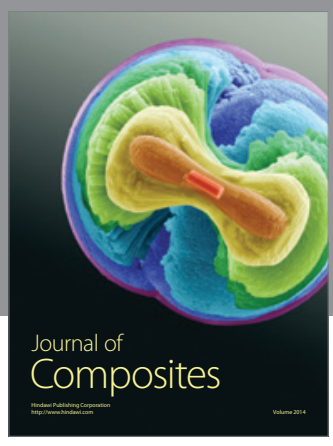
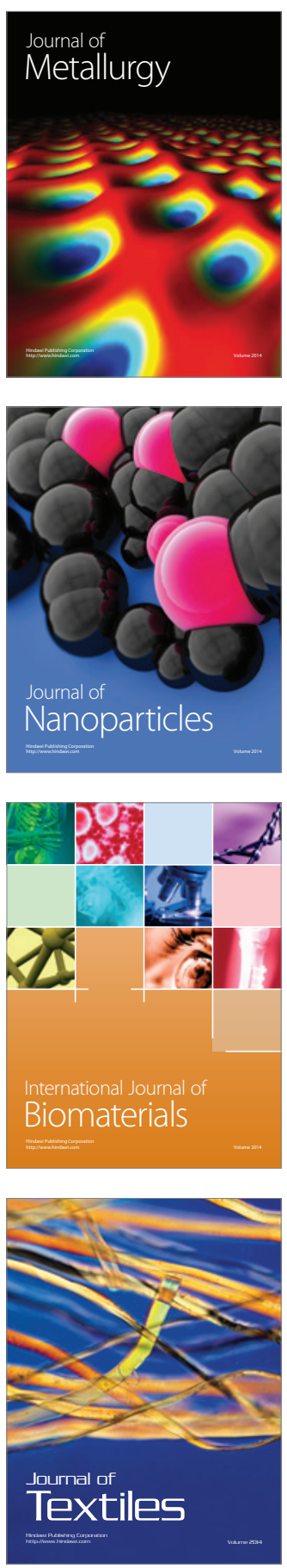